\title{
Trajectory planning of parallel mechanism for pouring robot
}

\author{
Long $\mathrm{Li}^{1,2}$, Chengjun Wang ${ }^{2, *}$ and Hongtao $\mathrm{Wu}^{1}$ \\ ${ }^{1}$ College of Mechanical and Electrical Engineering, Nanjing University of Aeronautics and Astronautics, Nanjing, 210016, China \\ ${ }^{2}$ College of Mechanical Engineering, Anhui University of Science and Technology, Huainan, 232001, China
}

\begin{abstract}
Aiming at the problem of singular area in the working space when designing the parallel casting mechanism of the pouring robot, and the sensitivity of the pouring liquid to acceleration of the ladle, we propose a genetic fusion algorithm of particle swarm optimization with angle and distance observers to find the optimal control point. Numerical analysis shows that it is feasible to change the tilting angle of the ladle to make it traverse the singular regions safely. According to the simplified method of single pendulum, the sloshing model of pouring liquid is established, and the segmented acceleration planning method considering the sloshing of pouring liquid is proposed in combination with the characteristics of high-speed cam motion. Numerical and experimental studies show that the segmented acceleration planning method can make the parallel pouring mechanism reach the set position in the shortest time while moving along the planned trajectory, and ensure that the sloshing of pouring liquid is within the safe range.
\end{abstract}

Keywords: Parallel mechanism, pouring robot, singularity, sloshing model, trajectory planning.

IN metal working, pouring is the process of transferring molten metal under high temperature from a ladle into a mould cavity. Because of its direct operation of high temperature molten metal, there are dust, vibration, noise and other harsh working environments, which poses a threat to the personal safety of workers, and various accidents are also common. In order to solve such problems, studies have been carried out on pouring robots, and various types of automatic pouring equipment have been developed for the pouring process ${ }^{1,2}$. The parallel pouring mechanism of the hybrid truss-type pouring robot ${ }^{3}$ is studied here, mainly for trajectory planning and acceleration planning.

At present, research on robot trajectory optimization is mostly concentrated on the series robots ${ }^{4-6}$; there are few studies on the trajectory planning of parallel mechanism, and most of them focus on several typical parallel platforms, such as DELTA and Stewart platform. Dasgupta et $a l .^{7}$ have designed an algorithm to select excellent path

\footnotetext{
*For correspondence. (e-mail: 270956876@163.com)
}

points and constructed continuous trajectory paths. Abdellatif and Heimann ${ }^{8}$ have proposed an adaptive algorithm for the shortest path of six-DOF (degree of freedom) manipulator. Experimental results show that the algorithm can give the required speed and corresponding driving force effectively. Pugazhenthi et al. ${ }^{9}$ proposed an algorithm for the Stewart platform which can maximize the structure stiffness and guarantee the workspace and singular constraints of the mechanism. Sen et al. ${ }^{10}$ used the variational method to design the non-singular path of parallel mechanism and to ensure that the actuator is within the specified length range. Afroum et al. ${ }^{11}$ parameterized the motion coordinates in the workspace of a three-DOF DELTA parallel robot, and used a continuous two-programming method to optimize the trajectory of the target position.

As early as 1996, Terashima et al. ${ }^{12}$ studied the sloshing of the ladle. The distribution parameter method of fluid was applied to the SOLA-MAC numerical simulation to describe the pouring process of the liquid. The result was used to guide the structure design of the ladle to better suppress the sloshing of the pouring liquid. Then a simplified pendulum model was proposed to make the design of the pouring-sloshing control system easier ${ }^{13}$. Hamaguchi et al. $^{14}$ studied the sloshing suppression of curved motion. Yamagata and Kaneko ${ }^{15}$ gave an optimized driving model to enable residual sloshing of the fluid to be better suppressed after rapid movement. Yano and Terashima ${ }^{16}$ used the $H^{\infty}$ model to suppress residual vibration of the ladle, and add a rotating controller to the active suppression of sloshing of the liquid in motion, with good results.

The above-mentioned studies only analyse the nonsingularity, stiffness, driving force and working time of several typical parallel mechanisms, and translation motion is considered more often, while few studies have been done on the coupling motion of rotation and translation. For the parallel pouring mechanism considered here, due to the structural and singular characteristics of the mechanism, there is a coupling relationship between rotation and translation. Previous studies have paid less attention to global acceleration time-varying sloshing suppression, and active suppression will increase the complexity of pouring systems. Therefore, this study proposes a fusion path optimization algorithm which gives 
(a)

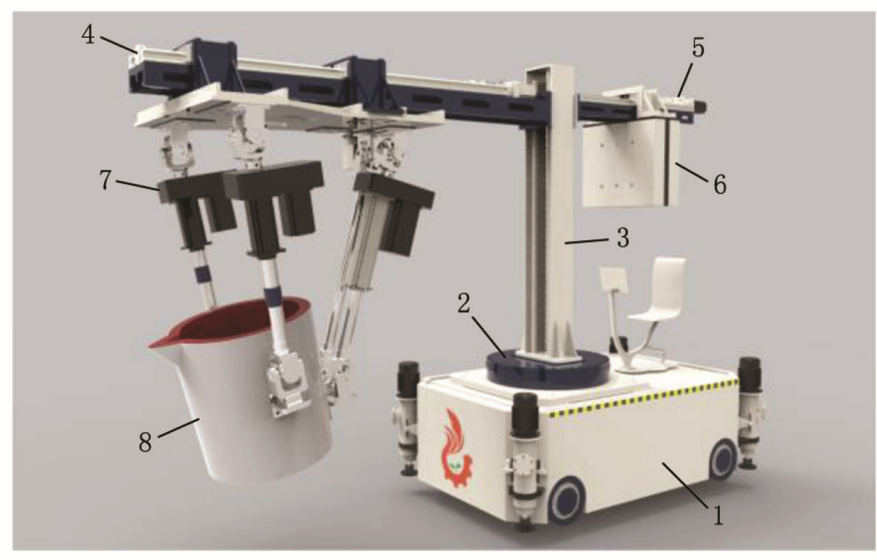

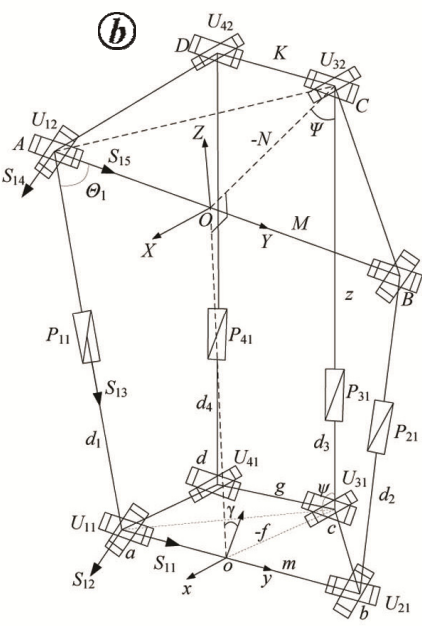

Figure 1. Schematic diagram of hybrid pouring robot and its parallel manipulator. $\boldsymbol{a}$, Hybrid truss-type movable pouring robot. 1, 4WD mobile platform; 2, Slewing device; 3, Hoisting device; 4, Forward device; 5, Backward device; 6, Counterweight device; 7, Parallel manipulator; 8, End-effector. $\boldsymbol{b}$, Four-UPU parallel manipulator of pouring robot.

the shortest path between any two points in the working space of the parallel pouring mechanism, and sets the angle observer and the safe distance observer to cross the singular regions by changing the pouring angle. Thereafter, the distribution scheme of acceleration and angular acceleration is given based on the sloshing model of pouring liquid and the motion model of high-speed cam, and the shortest operation time in the safe acceleration range is obtained.

\section{Mobile heavy load pouring robot}

\section{Structure of the casting robot}

Figure $1 a$ shows a hybrid truss-type mobile heavy load pouring robot $^{3,17}$. Since the ladle rotates around a certain axis during the pouring process and in order to adapt to the complex pouring environment, it is assumed that the ladle will not shake in other directions. Therefore, the parallel unit is designed to be a four-DOF parallel mechanism with one-axis rotation and three-axis translation. Based on the analysis of three-UPU parallel mechanism (three-DOF), a novel type of four-UPU parallel mechanism is proposed to meet the requirements of the pouring operation.

\section{Description of parallel pouring mechanism}

As shown in Figure $1 b$, the parallel manipulator of the heavy load pouring robot can be simplified as a four-UPU ( $\mathrm{U}$ indicating universal joint and $\mathrm{P}$ a shifting pair) parallel mechanism. The upper platform is fixed, installed in the heavy pouring robot beam, while the lower platform is in motion and is connected to the ladle. The four branches are UPU structural kinematic chains. At the time of initial assembly, the plane $A B a b$ (Figure $1 b$ ) formed by the chains 1 and 2 and the upper and lower platforms is vertical to the fixed platform $A B C D$, and the angle between the moving platform $a b c d$ and the plane $A B a b$ is $\gamma$. The plane $C D d c$ formed by the chains 3 and 4 and the upper and lower platforms, the angle between the fixed platform $A B C D$ and the plane $C D d c$ is $\psi$, while the angle between the moving platform $A B C D$ and the plane $C D d c$ is $\psi$. The fixed coordinate system $O-X Y Z$ is located at the midpoint of the $A B$ edge. The $Z$-axis is perpendicular to the fixed platform; and the $Y$-axis coincides with the $A B$ edge, point $C$ is on the extension of the $X$-axis. The moving coordinate system $o-x y z$ is located at the midpoint of the $a b$ edge. The $z$-axis is perpendicular to the moving platform and the $y$-axis coincides with the $a b$ edge. Point $c$ is on the extension of the $x$-axis and the $y$-axis is parallel to the $Y$-axis. The length of $o b$ is $m, o c$ is $f, d c$ is $g, O B$ is $M$, $O C$ is $N$ and $D C$ is $K$. The length of $A a, B b, C c$ and $D d$ is $d_{1}, d_{2}, d_{3}$ and $d_{4}$ respectively.

The $\mathrm{U}$ pair consists of $\mathrm{R}$ pairs ( $\mathrm{R}$ indicating rotating pair), whose two axes are perpendicular to each other, and the kinematic chain UPU is equivalent to the combination of the RRPRR joints. The axes of each chain kinematic pair are successively represented as $\boldsymbol{S}_{i 1}, \boldsymbol{S}_{i 2}, \boldsymbol{S}_{i 3}$, $\boldsymbol{S}_{i 4}$ and $\boldsymbol{S}_{i 5}(i=1,2,3,4$; for a simple diagram, only the axis of chain 1 is marked). $\boldsymbol{S}_{i 1}$ is consistent with the direction of $y$-axis and $\boldsymbol{S}_{i 5}$ is consistent with the direction of the $Y$-axis. The angle between $\boldsymbol{S}_{i 3}$ and $\boldsymbol{S}_{i 4}$ is $\Theta_{i}, \boldsymbol{S}_{i 1} \perp \boldsymbol{S}_{i 2}$, $\boldsymbol{S}_{i 4} \perp \boldsymbol{S}_{i 5}, \boldsymbol{S}_{12}\left\|\boldsymbol{S}_{22}, \boldsymbol{S}_{32}\right\| \boldsymbol{S}_{42}$.

\section{Singularity analysis of the 4-UPU parallel pouring mechanism}

The four-UPU parallel pouring mechanism comprises multiple closed chains, and the geometric constraint equation can be expressed by vector loop relation. 


$$
\begin{aligned}
& \boldsymbol{O} \boldsymbol{o}+{ }_{o}^{O} \boldsymbol{R} \boldsymbol{S}_{i 5}=\boldsymbol{S}_{i 1}+{ }_{i}^{O} \boldsymbol{R} \boldsymbol{S}_{i 3}, \\
& \dot{S}_{i 3}=\left({ }_{i}^{O} \boldsymbol{R} \boldsymbol{s}_{i 3}\right)^{T} \dot{\boldsymbol{O}} \boldsymbol{o}+\left({ }_{i}^{O} \boldsymbol{R} \boldsymbol{s}_{i 3}\right)^{T}{ }_{o}^{O} \boldsymbol{R} \tilde{\boldsymbol{\omega}}_{o} \boldsymbol{S}_{i 5}, \\
& \omega_{i}=\frac{1}{S_{i 3}} \tilde{\boldsymbol{s}}_{i 3}{ }_{i}^{O} \boldsymbol{R}^{T}\left(\dot{\boldsymbol{O}} \boldsymbol{o}+{ }_{o}^{O} \boldsymbol{R} \tilde{\boldsymbol{\omega}}_{o} \boldsymbol{S}_{i 5}\right) .
\end{aligned}
$$

In eq. (1), the subscripts of the rotation matrix represent transformation from the local coordinate system $o$ or $i$ $(i=1, \ldots, 4)$ to $O$ of the inertial coordinate system, $\boldsymbol{O o}=(o x, o y, o z)^{T} . \boldsymbol{s}_{i 3}$ is the unit vector of the axis along the $i 3$ moving pair, and $S_{i 3}$ represents the change in rod length of the $i 3$ moving pair. $\omega_{o}=(0, \psi, 0)$, and $\omega_{i}$ represents angular velocity of the branch $i . s_{45}$ represents the unit vector in the direction of the rotation drive axis. The Jacobian matrix of the four-UPU parallel pouring mechanism is

$$
\left[\begin{array}{l}
\dot{S}_{13} \\
\dot{S}_{23} \\
\dot{S}_{33} \\
\omega_{4}
\end{array}\right]=\boldsymbol{J a c o b}\left[\begin{array}{l}
\dot{\boldsymbol{O}} \boldsymbol{o} \\
\boldsymbol{\omega}_{o}
\end{array}\right]
$$

where

$$
\boldsymbol{J a c o b}=\left[\begin{array}{cc}
\left({ }_{1}^{O} \boldsymbol{R} \boldsymbol{s}_{13}\right)^{T} & \left({ }_{1}^{O} \boldsymbol{R} \boldsymbol{s}_{13}\right)^{T}{ }_{o}^{O} \boldsymbol{R} \tilde{\boldsymbol{S}}_{15}^{T} \\
\left({ }_{2}^{O} \boldsymbol{R} \boldsymbol{s}_{23}\right)^{T} & \left({ }_{2}^{O} \boldsymbol{R} \boldsymbol{s}_{23}\right)^{T}{ }_{o}^{O} \boldsymbol{R} \tilde{\boldsymbol{S}}_{25}^{T} \\
\left({ }_{3}^{O} \boldsymbol{R} \boldsymbol{s}_{33}\right)^{T} & \left({ }_{3}^{O} \boldsymbol{R} \boldsymbol{s}_{33}\right)^{T}{ }_{o}^{O} \boldsymbol{R} \tilde{\boldsymbol{S}}_{35}^{T} \\
\frac{1}{S_{43}} \boldsymbol{s}_{45}^{T} \tilde{\boldsymbol{s}}_{43}{ }_{4}^{O} \boldsymbol{R}^{T} & \frac{1}{S_{43}} \boldsymbol{s}_{45}^{T} \tilde{\boldsymbol{s}}_{43}{ }_{4}^{O} \boldsymbol{R}^{T}{ }_{o}^{O} \boldsymbol{R} \tilde{\boldsymbol{S}}_{45}^{T}
\end{array}\right] .
$$

$\boldsymbol{s}_{45}$ represents the unit vector of the axis of rotation.

The singularity of the mechanism can be analysed by taking the determinant of the Jacobian matrix as 0

$$
\operatorname{det}[\boldsymbol{J a c o b}]=0 \text {, }
$$

The parameters of the parallel pouring mechanism are substituted in eqs (5) and (6). Thus $s_{\mathrm{i} 3}=(0,0,-1)^{T}$, $\boldsymbol{s}_{45}=(0,1,0)^{T}$. The Jacobi singular equation of the mechanism is obtained as follows

$$
\begin{aligned}
& \frac{2 n \csc \psi \sec \psi(m-M) E F G}{d_{1} d_{2} d_{3} H}=0, \\
& E=n \sin \gamma+o z, \\
& F=N+o x-n \cos \gamma, \\
& G=o x \sin \gamma+o x \cos \gamma, \\
& H=n^{2}-2 n \cos \gamma(N+o x) \\
& \quad+2 n \cdot o z \sin \gamma+(N+o x)^{2}+o z^{2} .
\end{aligned}
$$

The singularity condition of the four-UPU parallel pouring mechanism can be obtained by eq. (7). We can conclude the following

(1) $m=M$ and $\psi=0^{\circ} / 90^{\circ}$, namely the fixed platform and moving platform are rectangular in the plane $A B b a$ and $C D d c$ of the parallel mechanism coincides or is perpendicular to the fixed platform; the determinant of Jacob is 0 . This kind of singularity is called structural singularity, and the four-UPU mechanism should be designed to avoid the same.

(2) When any one of $E, F, G$ and $H$ is 0 , the determinant of Jacob may be 0 . Further analysis shows that when the structure size is determined in eq. (7), the variables are $o x, o z$ and $\gamma$, indicating that the four-UPU parallel pouring mechanism is the singular of the position and attitude coupling of the $X Z$ plane in the workspace, and rewrite eq. (7)

$$
\begin{gathered}
\gamma_{H}=-\arctan \left(\frac{o z(N+o x)\left(n^{2}+(N+o x)^{2}+o z^{2}\right)-I}{n^{3} o z^{2}+(N+o x) I+n \cdot o z^{2}\left((N+o x)^{2}+o z^{2}\right.}\right) \\
I=\sqrt{-n^{2} o z^{2}\left(-n^{2}+(N+o x)^{2}+o z^{2}\right)}
\end{gathered}
$$

where $I$ in $\gamma_{H}$ has no solution in the real number domain. So $H$ is always not 0 , and we could consider the situation of $E \cdot F \cdot G=0$. Given the workspace and mechanism size: $-150 \mathrm{~mm} \leq$ ox $\leq-50, \quad-50 \mathrm{~mm} \leq$ oy $\leq-50, \quad-825 \mathrm{~mm} \leq$ $o z \leq-425,-28^{\circ} \leq \gamma \leq 62^{\circ} ; M=374 \mathrm{~mm}, N=680 \mathrm{~mm}$, $m=330 \mathrm{~mm}, f=365 \mathrm{~mm}$. Traversing the given workspace, we get the singular regions of $E \cdot F \cdot H=0$ (Figure 2). Figure 2 show the following.

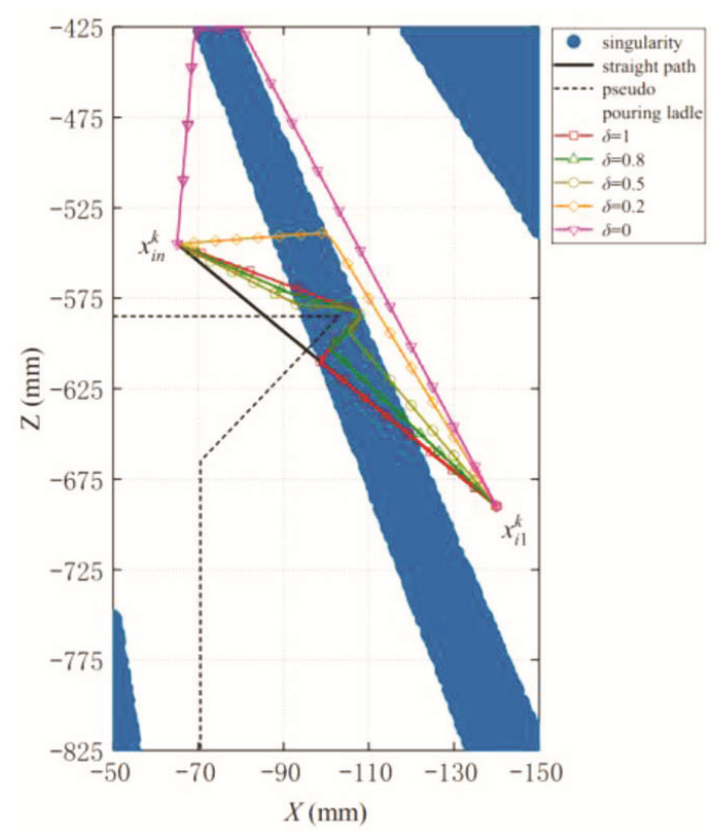

Figure 2. Path planning for singular regions and different weighting factors. 


\section{RESEARCH ARTICLES}

(1) The singular regions appear in the $X Z$ plane; there is no correlation with the $Y$-direction. Therefore, trajectory planning is only discussed in the $X Z$ plane, and the $Y$ direction method is the same.

(2) A part of the singular regions (inner area of the quadrangle in Figure 2) runs through the whole workspace; the singular point distribution is a plane of deflection, which shows that the singular angle $\gamma$ is continuously changing in the $X Z$ plane. Therefore, the $\gamma$ can be changed to make the ladle pass through the singular regions in a manner approaching the singular face. The other parts of the singular regions account for only about $10 \%$ of the workpace, and will not reach these regions when actually working so trajectory planning is not considered.

\section{Optimal trajectory planning based on a hybrid strategy}

\section{Particle swarm optimization algorithm}

In the $n$-dimensional search space, there are $p$ particles and the location of particle $i$ in the search space is expressed by vector $\boldsymbol{x}_{i}=\left[x_{i 1}, \ldots, x_{i n}\right]^{T} . G=G\left(\boldsymbol{x}_{i}\right)$ is the cost function of the specific problem, that is to evaluate the degree of fitness (optimization) of particle $i$ in the search space. Each particle will get an optimal solution through the search space, that is, $\boldsymbol{p} \boldsymbol{b}_{i}=\left[p b_{i 1}, \ldots, p b_{i n}\right]^{T}$. Particle swarm optimization (PSO) updates the global best location solution after each iteration, $\boldsymbol{g} \boldsymbol{b}=\left[g b_{i}, \ldots, g b_{n}\right]^{T}$. In the $k$ th iteration, the individual and global optimal location update processes are

$$
\begin{gathered}
\boldsymbol{p} \boldsymbol{b}_{i}^{k}= \begin{cases}\boldsymbol{p} \boldsymbol{b}_{i}^{k}, & \text { if } G\left(\boldsymbol{p} \boldsymbol{b}_{i}^{k-1}\right) \leq G\left(\boldsymbol{p} \boldsymbol{b}_{i}^{k}\right) \\
\boldsymbol{x}_{i}^{k}, & \text { if } G\left(\boldsymbol{p} \boldsymbol{b}_{i}^{k-1}>G\left(\boldsymbol{p} \boldsymbol{b}_{i}^{k}\right),\right.\end{cases} \\
\boldsymbol{g} \boldsymbol{b}^{i} \in\left\{\begin{array}{l}
\left(\boldsymbol{p} \boldsymbol{b}_{1}^{k}, \ldots, \boldsymbol{p} \boldsymbol{b}_{p}^{k}, \boldsymbol{g} \boldsymbol{b}^{i}\right) \mid G\left(\boldsymbol{g} \boldsymbol{b}^{i}\right)= \\
\min \left(G\left(\boldsymbol{p} \boldsymbol{b}_{1}^{k}\right), \ldots, G\left(\boldsymbol{p} \boldsymbol{b}_{1}^{k}\right), G\left(\boldsymbol{g} \boldsymbol{b}^{i-1}\right)\right)
\end{array}\right\} .
\end{gathered}
$$

In the iteration process, particle $i$ moves at the speed $\boldsymbol{v}_{i}=\left[v_{i 1}, \ldots, v_{i n}\right]^{T}$ in the search space. The velocity and position of each particle are corrected as follows

$$
\begin{aligned}
& v_{i j}^{k+1}=w v_{i j}^{k}+c_{1} \operatorname{rand}_{2}()\left(p b_{i j}^{k}-x_{i j}^{k}\right)+c_{2} \operatorname{rand}_{2}()\left(g b_{j}^{k}-x_{i j}^{k}\right), \\
& x_{i j}^{i+1}=x_{i j}^{i+1}+v_{i j}^{k+1} .
\end{aligned}
$$

In eqs (11) and (12), $j=1, \ldots, n . c_{1}$ and $c_{2}$ are normal numbers, which are called acceleration factors. rand $_{1}$ and $\operatorname{rand}_{2}$ are normal distribution functions between $[0,1] ; w$ is the inertia factor and usually ranges from 0.1 to 0.9 , and larger the value, stronger is the global search ability. Conversely, the smaller the values of $w$, the stronger the local search ability.

In order to avoid the premature of the PSO algorithm, this study proposes an improved PSO algorithm based on point-to-point of trajectory planning, and an adaptive strategy is used to modify the particle moving velocity using the orientation vectors of trajectory planning starting point and end point. That is

$$
\begin{aligned}
& v_{i j}^{k+1}=w v_{i j}^{k}+c_{1} \operatorname{rand}_{2}() \Delta v_{i j}^{k 1}+c_{2} \operatorname{rand}_{2}() \Delta v_{i j}^{k 1}, \\
& \left\{\begin{array}{l}
\Delta v_{i j}^{k 1}=\Delta_{i j}^{k 1}\left(p b_{i j}^{k}-x_{i j}^{k}\right), \\
\Delta v_{i j}^{k 2}=\Delta_{i j}^{k 2}\left(g b_{j}^{k}-x_{i j}^{k}\right) .
\end{array}\right.
\end{aligned}
$$

Revised $\Delta v_{i j}^{k 1}$ and $\Delta_{i j}^{k 2}$ are as follows

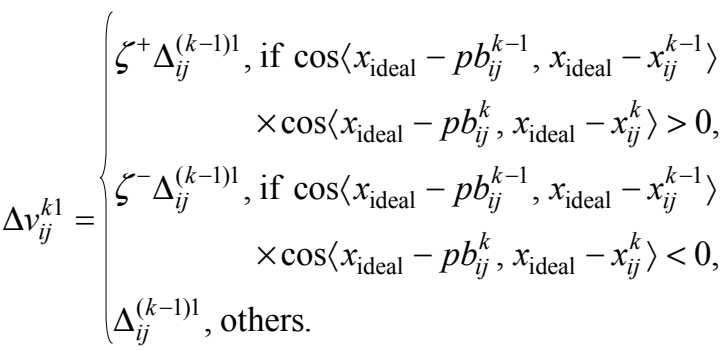

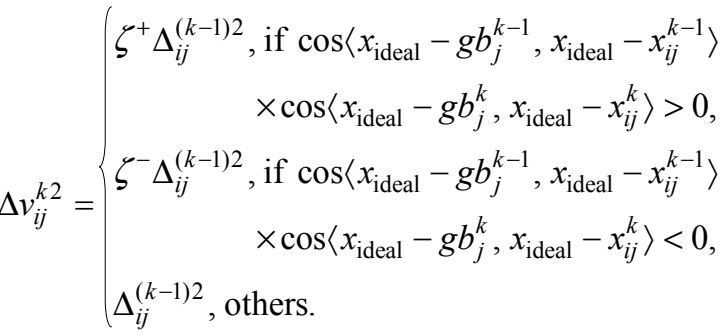

In eqs (15) and (16), $x_{\text {ideal }}$ represents the ideal location vector of the starting point to the end point, and $\cos \langle$, represents the cosine of the direction vector.

The ideal position vector from the starting point to the end point is used as the target direction vector, in eqs (15) and (16), when the product of the two cosines of a particle is greater than zero, it indicates that the particle is approaching the optimal solution of the individual (global) from one side. At this time, it can increase $\Delta_{i j}^{(k-1) 1}\left(\Delta_{i j}^{(k-1) 2}\right)$ to speed up the convergence rate of the algorithm and take a multiplier $\zeta^{+}$; if the product of the two cosines of the particle is less than zero, it shows that the particle is hovering near the individual (global) extremum; then $\zeta^{-}$can be reduced to avoid particles lingering around the extremum and resulting in a premature algorithm. If the product of the two cosines of the particle is zero, then $\Delta_{i j}^{(k-1) 1}\left(\Delta_{i j}^{(k-1) 2}\right)$ does not change, $0<\zeta^{-}<$ $1<\zeta^{+}$. 


\section{Angle observer}

By the analysis of $E, F$ and $H$, the singularity of fourUPU is related to $o x, o z$ and $\gamma$. In order to cross the singular regions, the strategy of changing $\gamma$ is adopted; that is, by changing the tilting angle of ladle, the $E \cdot F \cdot G \neq 0$ will be ensured such that the parallel pouring mechanism passes through the singular region smoothly. The specific search process is as follows:

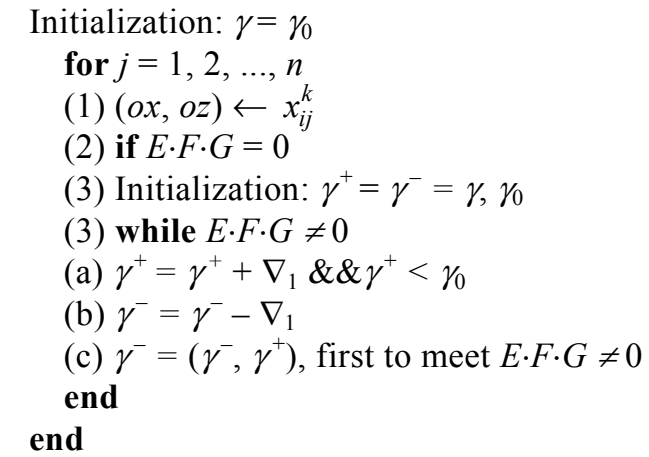

where $\gamma_{0}$ is the titling angle of the ladle during the initial movement. In the above search process, the dip angle searches for the minimum increase (reduction) of $\nabla_{1}$ in two directions. However, the increase cannot exceed the initial tilt angle of $\gamma_{0}$, because in the actual operation, exceeding $\gamma_{0}$ will cause splashing of the ladle and thus accidents.

\section{Distance observer}

Combined with the actual working environment analysis, the most common type of collision is that between the ladle and the pouring cup. In the method used here, the distance $r$ between the centre of the ladle and the edge of the pouring cup is the minimum safe distance (Figure 3 ). The specific search process is as follows

$$
\begin{aligned}
& \text { for } j=1,2, \ldots, n \\
& \text { (1) }(o x, o z) \leftarrow x_{i j}^{k} \\
& \text { (2) look for } p c x_{\min }, p c z_{\min } \\
& \text { (2) if }\left(o x-p c x_{\min }\right)^{2}-\left(o z-p c z_{\min }\right)^{2} \geq r^{2} \\
& \text { (3) while }\left(o x-p c x_{\min }\right)^{2}-\left(o z-p c z_{\min }\right)^{2}<r^{2} \\
& \text { (a) } o x=o x+\nabla_{2} \\
& \text { (b) } o z=p c z_{\min }-\frac{o z_{0}-p c z_{\min }}{o x_{0}}\left(p c x_{\min }-o x\right) \\
& \text { end } \\
& \text { (4) } x_{i j}^{k} \leftarrow(o x, o z) \\
& \text { end }
\end{aligned}
$$

$p c x_{\min }, p c z_{\min }$ is the nearest point of the tilting centre of the ladle and the edge of the pouring cup. The positive and negative values of the increment $\nabla_{2}$ depend on the direction of movement of the ladle.

\section{GA-IPSO algorithm}

Figure 4 is a flow chart of the genetic algorithm-improve particle Swarm optimization (GA-IPSO) algorithm.

Random dimensionality reduction: A large number of control points are necessary to fit the path. The efficiency of the algorithm will be reduced, and so the dimension of individual position vectors is reduced randomly to improve the efficiency of the algorithm. The specific process is as follows

$$
\left[x_{i 1}^{k}, x_{i 2}^{k}, \ldots, x_{i n}^{k}\right]^{T} \underset{\left[x_{i 1_{1}}^{k}, \ldots, x_{i n_{2}}^{k}\right]^{T}}{\stackrel{\text { Random }}{\longrightarrow}}\left[x_{i 1}^{k}, \ldots, x_{i\left(n-\left(n_{2}-n_{1}+1\right)\right)}^{k}\right]^{T} .
$$

In eq. (17), the $n_{1}-n_{2}$ positions are randomly deleted from the position vector of the individual, and the remaining $n-\left(n_{2}-n_{1}+1\right)$ dimensional position vector recombination continues to solve the algorithm.

\section{Numerical analysis}

(a) Size and layout of parallel pouring mechanism and pouring cup: The parallel pouring mechanism adopts the design size, workspace and singular regions mentioned earlier in the text in the section on singularity analysis. The size of the gate cup was selected according to Pan and $\mathrm{Xu}^{18}$. In order to have both distance collision and singular path planning, the layout shown in Figure 3 is adopted.

(b) Cost function: The cost function is defined as

$$
G=\delta \psi\left(p b_{i}^{k}\right)+(1-\delta) \varphi\left(p b_{i}^{k}\right)
$$

In eq. (18), $\psi\left(\boldsymbol{p} \boldsymbol{b}_{i}^{k}\right)$ represents the $k$ th iteration path length (step number) of particle $i$, and $\varphi\left(\boldsymbol{p} \boldsymbol{b}_{i}^{k}\right)$ represents

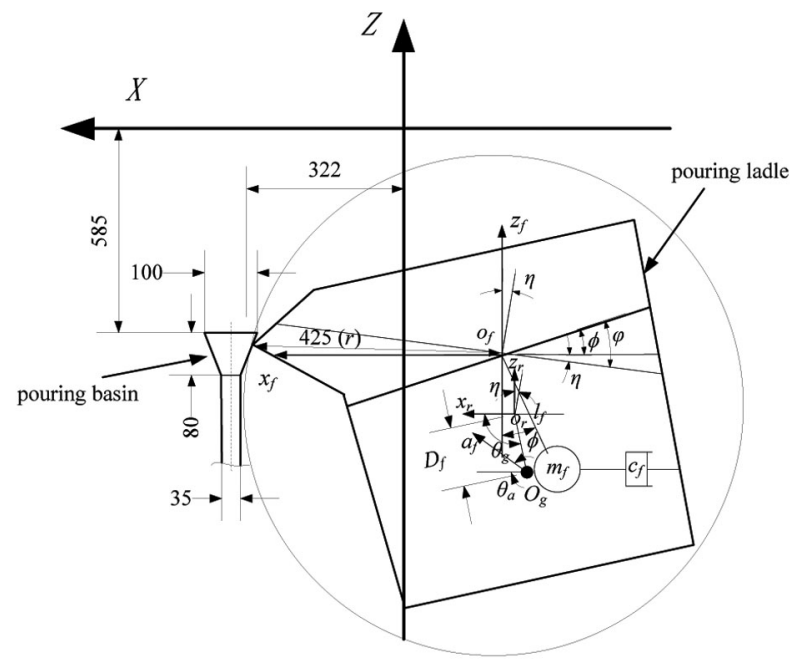

Figure 3. The size, layout and pendulum sloshing model of the ladle 
the step number of angle change in the $k$ th iteration path of particle $i$. The algorithm takes the sum of steps of distance and the angle as the cost function. $\delta$ is a weight coefficient in the range $0 \leq \delta \leq 1 . \delta=0$ represents the minimum angle problem, while $\delta=1$ represents the minimum path problem.

(c) Algorithm assignment: In order to ensure appropriate speed of maturation, $w, r_{1}$ and $r_{2}$ in eq. (13) are 0.6, 1.2 and 1.2 respectively, $\zeta^{+}$and $\zeta^{-}$are 0.8 and 0.2 respectively, and initial $\Delta_{i j}^{11}$ and $\Delta_{i j}^{21}$ are 1 . The variation rate $p_{m}$ and the cross rate $p_{\mathrm{c}}$ are 0.2 and 0.5 respectively. The initial position and target position of the moving platform are $x_{i n}^{k}=[-140,-690]$ and $x_{i n}^{k}=[-65$ and -545] respectively, and the angle of initial tilting is $\gamma=20^{\circ}$. The number of particles $p=40$. The algorithm terminates under the following conditions: (1) maximum number of iterations is 1000 times, or (2) in two successive iterations, fitness of the best particles is less than 1. The incremental $\nabla_{1}$ of the distance observer and incremental $\nabla_{2}$ of the angle observer are $1 \mathrm{~mm}$ and $0.1^{\circ}$ respectively, smaller $\nabla_{2}$ values can ensure correctness and continuity of angle-fitting. The minimum security distance needs to be properly amplified and take $r=430 \mathrm{~mm}$.

Due to the acceleration method and the quality requirements of algorithm in the singular regions, the number of initial control points (dimension of the position vector) is 180 , and the number of the minimum control points (dimension of the position vector) is 60 . The algorithm is compiled into MATLAB, and the control points are all rounded. The result is fitted by a simple B spline curve.

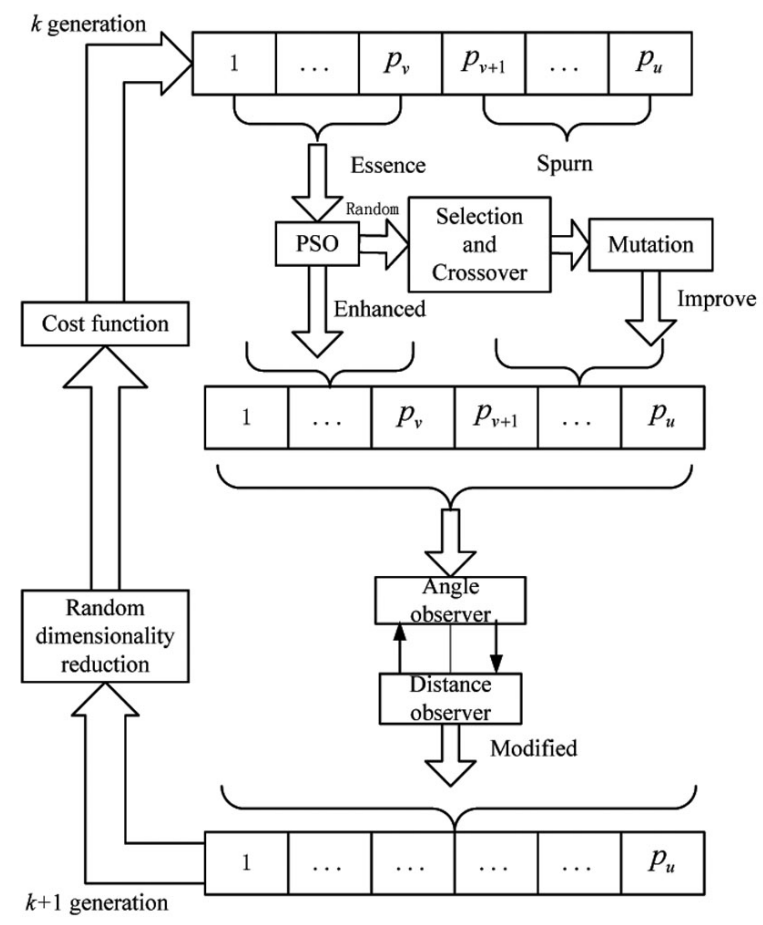

Figure 4. Flow chart of the GA-PSO algorithm.
Table 1 shows a comparison of different $\delta$ values in GA-IPSO algorithm for path planning. The simulations were run on a Xeon E5 $2.10 \mathrm{GHz}$ computer and the average of 10 simulation results was taken. The computing time is directly chosen by MATLAB's own 'cputime' function. The range of the $\delta=0-1$, where $\delta=0$ is equivalent to the minimum rotation angle of the ladle, while the $\delta=1$ is equivalent to the shortest path problem. The path planning results corresponding to different weighting factors in Table 1 are shown in Figures 2 and 5. In which Figure $5 a$ and $b$, in order to clearly show the angle change in the path planning, $\gamma-Z$ and $\gamma-X$ are used as coordinates respectively.

Table 1 shows that with the decrease of $\delta$, the number of iterations, calculation time and fitness are significantly improved. Combined with Figures 2 and 5, it can be seen that this is due to increase in the weight factor of the ladle angle, which leads to a large deviation in the trajectory of the ladle from the pouring cup; the search process of the singular regions and rotation angle of the ladle are shortened; the correction times of the distance observer and angle observer decrease, thus accelerating the convergence of iteration. However, the $\delta=0$ and $\delta=0.2$ obviously are not the desired trajectories, because they deviate from the ideal trajectory (straight path). When $\delta=1$, the number of iterations, calculation time and fitness value are the largest, and the algorithm efficiency is poor. When $\delta=0.8$ and $\delta=0.5$, the planned paths are next to each other, and the calculation time and fitness value of the algorithm are also close and moderate. Therefore, $\delta=0.5-0.5$ is selected as the best compromise for the two competitive targets in the GA-IPSO algorithm (trajectory and ladle rotation angle).

Table 2 shows a comparison between standard PSO algorithm and GA-IPSO algorithm for $\delta=0.5$ and particle number $p=40$. It can be seen from the table that the GA-IPSO algorithm is not only efficient in computation but also in the quality of the final value compared with the standard PSO algorithm.

Table 1. Performance analysis of planned trajectories for various weighting factors $(\delta)$

\begin{tabular}{lrrrrr}
\hline No. of particles & \multicolumn{7}{l}{40} \\
\hline$\delta$ & 1 & 0.8 & 0.5 & 0.2 & 0 \\
No. of iteration & 553 & 488 & 413 & 387 & 321 \\
Computation time (s) & 1182 & 1022 & 926 & 774 & 664 \\
Fitness value & 178 & 149 & 132 & 110 & 80 \\
\hline
\end{tabular}

Table 2. Comparison of standard PSO algorithm and GA-IPSO for weighting factor $\delta=0.5$

\begin{tabular}{lcc}
\hline Optimization & Standard PSO & GA-IPSO \\
\hline No. of iteration & 791 & 413 \\
Computation time (s) & 1673 & 926 \\
Fitness value & 141 & 132 \\
\hline
\end{tabular}



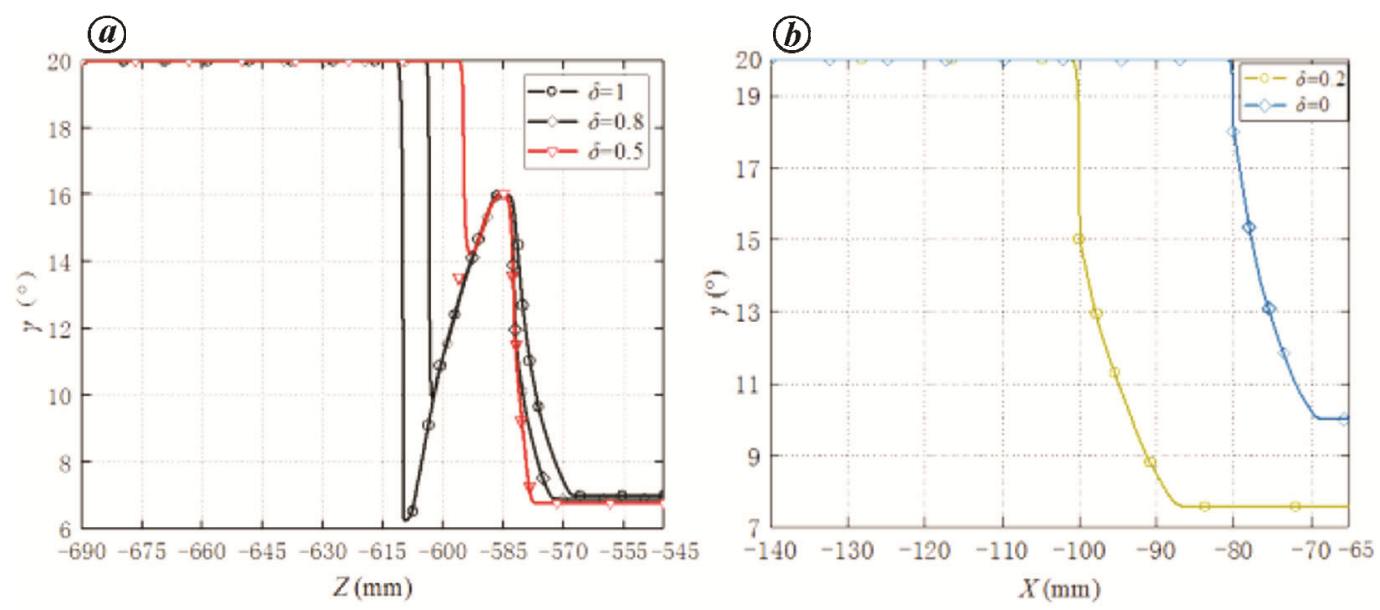

Figure 5. Variation of ladle inclination angle. $\boldsymbol{a}, \delta=1, \delta=0.8$ and $\delta=0.2 ; \boldsymbol{b}, \delta=0.2$ and $\delta=0$.

\section{Acceleration planning considering sloshing of pouring liquid}

\section{Pendulum-type model}

In order to facilitate the combination of sloshing model and acceleration model of the trajectory, we use the pendulum method to establish the sloshing model of the pouring liquid (Figure 3).

In Figure 3, $o_{f}-x_{f}-z_{f}$ is a liquid surface coordinate system, $\overline{o_{f} x_{f}}$ is parallel to the horizontal plane, $o_{r}-\underline{x_{r}-z_{r}}$ is located in the rotating fulcrum coordinate system, $\overline{o_{r} x_{r}}$ is parallel to the horizontal plane, $m_{f}$ the quality of pouring liquid, $c_{f}$ the equivalent damping, $l_{f}$ the equivalent pendulum length, $\eta$ the pouring angle, $\varphi$ the sloshing angle of the pouring liquid, $\phi(\phi=\eta+\varphi)$ the sloshing angle relative to the horizontal plane, $o_{r}$ the centre of rotation, $o_{g}$ the centre of mass of the pouring liquid, $D_{f}$ the distance between $o_{r}$ and $o_{g}, o_{f}$ the pivot of the pendulum, $\theta_{g}$ the angle between $\overline{o_{r} o_{g}}$ and the horizontal, and $\theta_{g}$ the angle between the acceleration $a_{f}$ and the horizontal, $J$ is the moment of inertia, $J=m_{f} l_{f}^{2}$. The pendulum sloshing equation of the pouring liquid at point $o_{f}$ can be written as

$$
\begin{aligned}
& J \frac{\mathrm{d}^{2}(\eta+\varphi)}{\mathrm{d} t^{2}}=-c_{f} \frac{\mathrm{d}\left\{l_{f}(\eta+\varphi)\right\}}{\mathrm{d} t} l_{f} \cos (\eta+\varphi) \\
& -m_{f} g l_{f} \sin (\eta+\varphi)-m_{f} \frac{\mathrm{d}\left\{\left(D_{f} \cos \theta_{g}\right) \eta\right\}}{\mathrm{d} t^{2}} l_{f} \sin (\eta+\varphi) \\
& -m_{f} \frac{\mathrm{d}\left\{\left(D_{f} \sin \theta_{\mathrm{g}}\right) \eta\right\}}{\mathrm{d} t^{2}} l_{f} \cos (\eta+\varphi) \\
& -m_{f} a \cos \left(\theta_{a}\right) l_{f} \cos (\eta+\varphi) \\
& +m_{f} a \sin \left(\theta_{a}\right) l_{f} \sin (\eta+\varphi) .
\end{aligned}
$$

For $\phi=\eta+\varphi$, due to the small amplitude of sloshing of the pouring liquid, linearization of eq. (19) can be obtained directly.

$$
\ddot{\phi}=-\frac{c_{f}}{m_{f}} \dot{\phi}-\frac{g}{l_{f}} \phi-\frac{D_{f} \sin \theta_{g}}{l_{f}} \ddot{\eta}-\frac{a_{f} \cos \theta_{d}}{l_{f}} .
$$

By the pendulum characteristic, we can get the natural frequency $\omega_{n}$ and the damping coefficient $\zeta$

$$
\omega_{n}=\sqrt{\frac{g}{l_{f}}}, \zeta=\frac{c_{f}}{2 m} \sqrt{\frac{l_{f}}{g}} .
$$

$\omega_{n}$ and $\zeta$ can be easily measured by experiments, or can be estimated by eqs $(22)$ and $(23)^{19,20}$.

$$
\begin{aligned}
& \zeta=d_{1} v^{1 / 2}\left(B_{1} / 2\right)^{-3 / 4} g^{-1 / 4}, \\
& L_{f} / l_{f} \approx 2-3 .
\end{aligned}
$$

where $d_{1}$ is the depth of liquid, $v$ the viscosity of liquid, $B_{1}$ the average width of the ladle and $L_{f}$ is the distance between $o_{r}$ and $o_{f}$.

\section{Acceleration planning}

The acceleration planning requirements under the condition of sloshing are proposed referring to the motion law of high speed $\mathrm{cam}^{21}$. (1) The speed and acceleration of the parallel pouring mechanism at starting, stopping and the fold points of the polyline in the planned trajectory are all 0. (2) There is no jerk when the ladle is stopped. (3) Since the partial translational motion is accompanied by rotation of the ladle, in order to reduce acceleration variation of the composite motion, the pure translational motion time: translational + rotational motion time is $3: 7$. 
The boundary conditions are according to the requirements

$$
\begin{aligned}
& s_{t 0}(0)=v_{t 0}(0)=a_{t 0}(0)=0, \\
& s_{t n}(1)=1 \\
& v_{t n}(0)=a_{t n}(0)=j_{t n}(0)=0, \\
& t_{t T}: t_{t T R}=3: 7
\end{aligned}
$$

Additional constraints

$$
\left\{\begin{array}{c}
s_{t 2}\left(t_{2}\right)=l_{t 2}, v_{t 2}\left(t_{2}\right)=a_{t 2}\left(t_{2}\right)=0 \\
s_{t 3}\left(t_{3}\right)=l_{t 3}, v_{t 3}\left(t_{3}\right)=a_{t 2}\left(t_{2}\right)=0 . \\
\cdots
\end{array}\right.
$$

In eqs (24)-(28), $s_{t i}, v_{t i}, a_{t i}$ and $j_{t i}$ are path length, speed, acceleration and jerk $(i=0,1,2, \ldots, n)$ respectively. $t_{t T}$ and $t_{t T R}$ are translation time and compound motion time respectively, and the displacement function is $n-1$ order polynomial.

$$
s_{t}=\rho_{0}+\rho_{1} t+\rho_{2} t^{2}+\cdots+\rho_{n-1} t^{n-1} .
$$

In eq (29), $\rho_{0}, \rho_{1}, \rho_{2}, \ldots, \rho_{n-1}$ are coefficients of polynomials. According to the equation, we can get $v_{t}, a_{t}$ and $j_{t}$ by continuous derivation.

Translation path acceleration planning: Translation is the main movement of the ladle, and so it is the emphasis of acceleration planning. Let us assign the model in Figure 3 to the actual operating conditions, $d_{1}=0.37 \mathrm{~m}$, $v=1.0067 \times 10^{-6} \mathrm{~m}^{2} / \mathrm{s}$. The upper and lower diameters of the inner cavity of the ladle are 300.52 and $0.47 \mathrm{~m}$ respectively, and height is $0.54 \mathrm{~m} . B_{1}=0.486 \mathrm{~m}$, $\zeta=6.06 \times 10^{-4} . L_{f} / l_{f}$ takes an average of $2.5, L_{f}=0.1 \mathrm{~m}$, and $l_{f}=0.04 \mathrm{~m} . D_{f}=0.085 \mathrm{~m}, \theta_{g}=-1.919 \mathrm{rad}$. At the initial time, $\phi(0)=0, \phi^{\prime}(0)=0$. The preferred path and angle parameters are obtained by taking $\delta=0.5$.

(a) Global acceleration planning: The normal acceleration planning is used to plan the whole path. When $\delta=0.5$, path length is $0.174 \mathrm{~m}$. Since the planned trajectory is approximately a polyline, the lengths of the straight segments between the fold points are $0.103 \mathrm{~m}$, $0.014 \mathrm{~m}, 0.016 \mathrm{~m}$ and $0.044 \mathrm{~m}$ respectively, starting from the starting point of the trajectory. According to the acceleration plan proposed here we can get

$$
\begin{aligned}
& s_{t 0}(0)=v_{t 0}(0)=a_{t 0}(0)=0, \\
& s_{t 5}(1)=1, \\
& v_{t 5}(0)=a_{t 5}(0)=j_{t 5}(0)=0 .
\end{aligned}
$$

Additional constraints

$$
\left\{\begin{array}{l}
s_{t 2}(0.569)=0.592, v_{t 2}(0.569)=a_{t 2}(0.569)=0 \\
s_{t 3}(0.683)=0.655, v_{t 3}(0.683)=a_{t 2}(0.683)=0 \\
s_{t 5}(0.849)=0.747, v_{t 3}(0.849)=a_{t 2}(0.849)=0
\end{array}\right.
$$

Equations (30)-(33) are introduced into MATLAB and the global acceleration is obtained. Figure 6 shows the change in acceleration in unit time. The global acceleration planning scheme has the following disadvantages:

(i) The peak acceleration is extremely large; it will increase the burden of the drive unit of the parallel mechanism. The acceleration in the first half of the time changes drastically, and the acceleration change in the latter half of the time is basically zero, and the full time is not fully utilized.

(ii) The highest power of the fitting polynomial is 13 , increasing the calculation time.

$$
T_{\text {total }}=\sqrt{\frac{A_{\max } \times S_{\text {total }}}{a_{\text {max }}}} .
$$

In eq. (34), $A_{\max }$ is the maximum acceleration in unit time. $S_{\text {total }}$ is the total length of a given path, $a_{\max }$ the maximum acceleration of a given path, and $T_{\text {total }}$ is the total time required to traverse the path at a given $a_{\max }$.

(b) Piecewise acceleration planning: In view of the sensitivity of the pouring liquid to impact vibration, a piecewise planning method with breaking points as the starting point and ending point is proposed.

$$
\left\{\begin{array}{l}
s_{t e 0}(0)=0, v_{t e 0}(0)=a_{t e 2}(0)=0, \\
a_{t e 2}(0.3)=0, s_{t e 3}(1)=1, \\
v_{t e 3}(1)=a_{t e 3}(1)=j_{t e 3}(1)=0 .
\end{array}\right.
$$

In eq. (35), $e=1,2,3,4$. In the pouring ladle movement, it is assumed that the pouring liquid will have longer sloshing buffer time in each part, so as to better adapt to the next acceleration change, and make $a_{t e 2}(0.3)=0$. Equation (35) is introduced into MATLAB to get the acceleration of each segment

$$
\begin{aligned}
& a_{t e}=1235.294 t^{5}-3829.412 t^{4} \\
& +4249.412 t^{3}-1951.765 t^{2}+296.471 t, \\
& A_{\max \_e}=14.112 .
\end{aligned}
$$

In eq. (37), $A_{\max _{e} e}$ represents the maximum acceleration of unit time within each planning segment of the path. Figure 6 is the variation of acceleration in unit time during piecewise acceleration planning. It can be seen that in the actual acceleration fitting, the unit acceleration peak 
of the piecewise acceleration planning is very small; the burden of the drive unit of the parallel mechanism is less. The time required for each segment of acceleration to reach the peak is very short, thereafter, the change in acceleration is slow, which makes full use of the whole time. The highest power of the fitting polynomial of acceleration is 8 , and the calculation speed is fast.

Therefore, in this study, a piecewise acceleration planning strategy is applied to the acceleration planning of the translational path. The actual acceleration $a_{t e} A$ of each segment is:

$$
\begin{aligned}
& a_{t e_{-} A}=\frac{a_{\max }}{A_{\max }}\left(\begin{array}{l}
1235.294 \tau^{5}-3929.412 \tau^{4}+4249.412 \tau^{3} \\
-1951.765 \tau^{2}+296.471 \tau
\end{array}\right), \\
& \tau=\frac{t}{T_{\text {total }}} .
\end{aligned}
$$

Rotational acceleration planning: The rotational acceleration is also divided by piecewise planning. In Figure 5 , the change in tilting angle is one-to-one correspondence with the change in translation path. Therefore, the corresponding relations between rotation and translation in Figure 5 can be directly used for polynomial fitting.

\section{Experimental verification}

First, we use the acceleration planning to plan acceleration of the path. At $\delta=0.5, \theta_{a}$ of the ladle is $1.221,1.954$, 0.139 and $0.872 \mathrm{rad}$. Further analysis of eq. (20) shows that $D_{f} \sin \theta_{g} / l_{f}=0.725$ and $\cos \theta_{a} / l_{f(\min )}=8.565$, which indicates that rotational acceleration has little effect on sloshing. In addition, the prototype is still in the design and processing stage. Therefore, the validation experiment only makes acceleration planning for translation path.

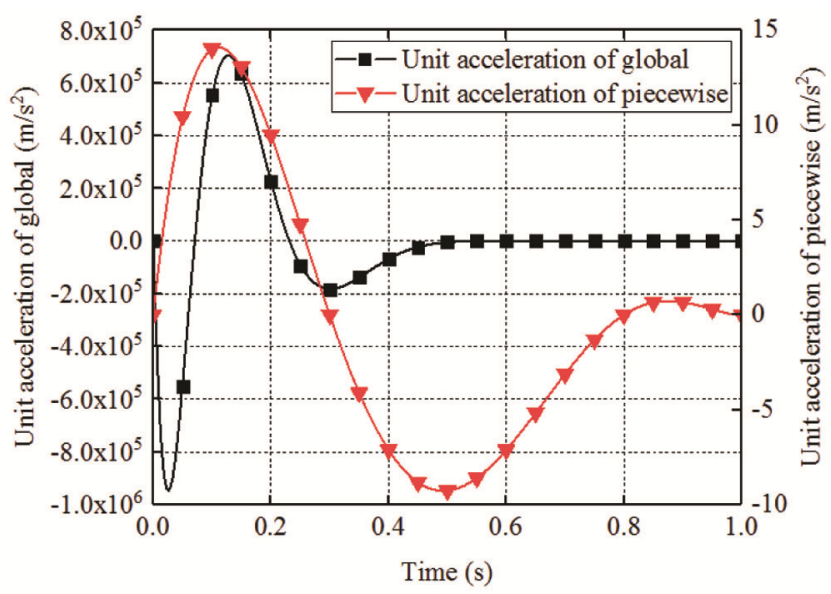

Figure 6. Unit acceleration of global acceleration planning and piecewise acceleration planning.
The verification experiment was performed on a six-DOF series robot. Since the kinematic viscosity of water is similar to that of molten cast iron and is readily available in the laboratory, water was selected as the study fluid. Figure 7 shows the experimental set-up. The experiment mainly studies sloshing of the liquid in the container under translational state. The sloshing angle of the liquid is measured as shown in Figure 7. Sloshing angle is

$$
\phi=\arctan \frac{\left(d p_{2}-d p_{1}\right)}{B_{p} / 2} .
$$

In eq. (39), $d p_{1}$ and $d p_{2}$ are liquid-level heights, and $B_{p}$ is the distance between the liquid level sensors. The trajectory of the container moves along the preferred path at $\delta=0.5$. The sloshing law of the liquid in the container and acceleration change of the end of the robot are obtained by the pendulum model and piecewise acceleration model respectively. The maximum acceleration at each stage is calculated to be $1.39,1.07,0.24$ and $0.36 \mathrm{~m} / \mathrm{s}^{2}$, and maximum sloshing angle is $0.08 \mathrm{rad}$. Figure $8 a$ shows numerical simulation and experimental results.

Comparing the numerical simulation results and experimental results in Figure $8 a: 0-2.91 \mathrm{~s}$ is the sloshing state of water in the container during the robot movement time, and 2.91-5 $\mathrm{s}$ is the free sloshing of the container at the target position. The sloshing amplitude obtained by the experiment in $0-5 \mathrm{~s}$ is slightly attenuated because the viscous resistance of water and the wall of the container are not considered in the model. However, the form and frequency of sloshing are basically the same, and the sloshing angle does not exceed the maximum threshold. This indicates that the liquid sloshing and acceleration planning methods have higher operating efficiency and can prevent splashing accidents caused by severe sloshing. Figure $8 b$ shows the instantaneous sloshing state of

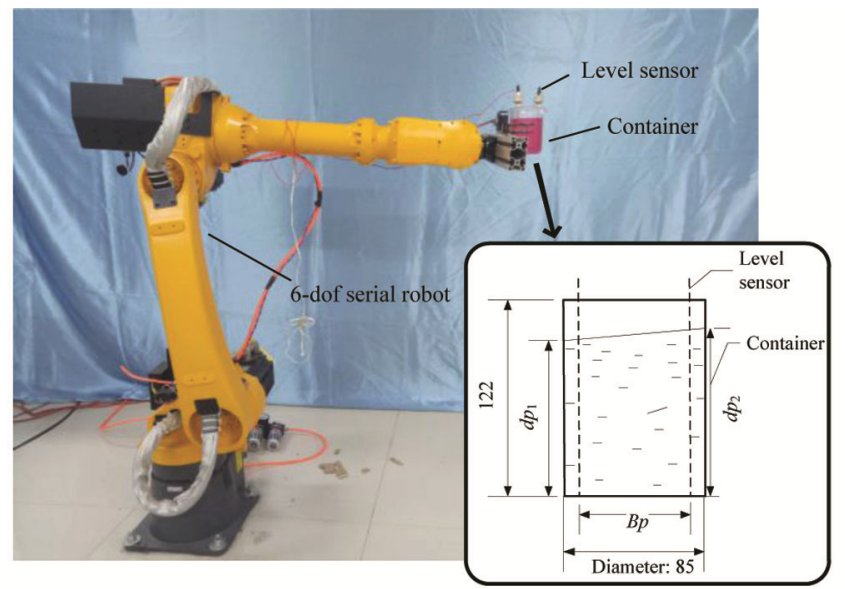

Figure 7. Experimental device for liquid sloshing and its measurement method. 


\section{RESEARCH ARTICLES}

(b)

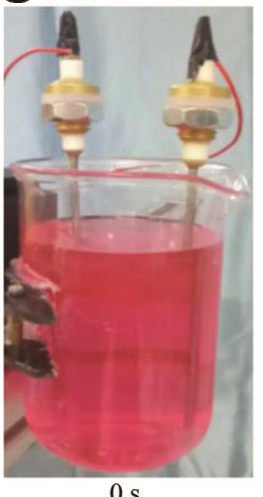

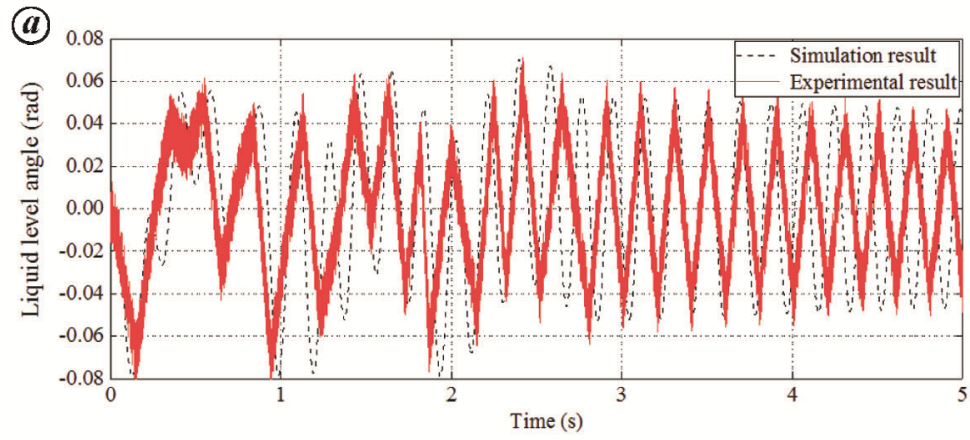

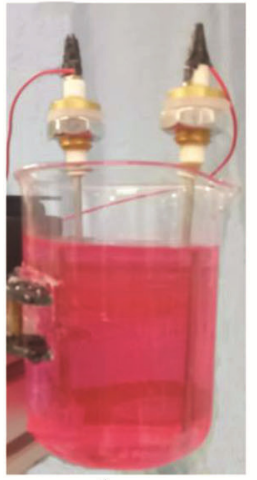

$1 \mathrm{~s}$

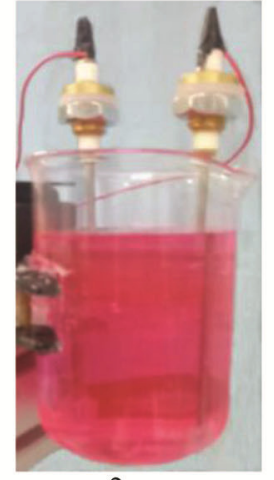

$2 \mathrm{~s}$

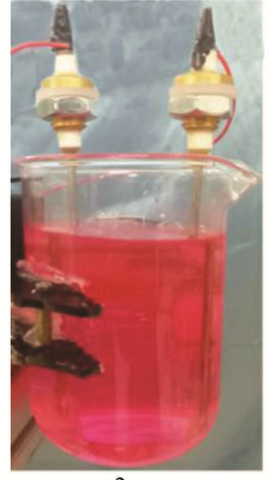

$3 \mathrm{~s}$

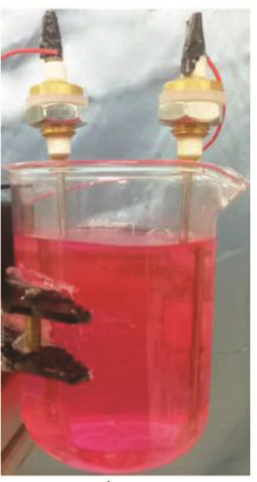

$4 \mathrm{~s}$

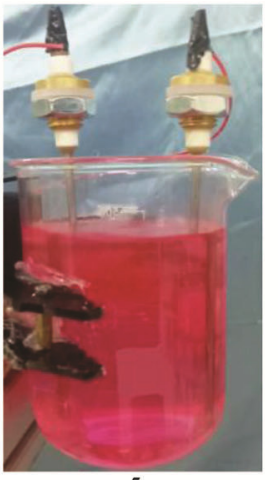

$5 \mathrm{~s}$

Figure 8. Simulation and experimental results of liquid sloshing. $\boldsymbol{a}$, Simulation and experimental results of liquid sloshing at $\delta=0.5$. $\boldsymbol{b}$, Instantaneous sloshing of liquid in the experiment.

water in the container, corresponding to the instantaneous sloshing amplitude in Figure $8 a$, and the instantaneous liquid-level sloshing is stable without violent fluctuation.

\section{Conclusion}

Here, a four-UPU parallel pouring mechanism with fourDOF for casting robot is studied, and a path planning method and acceleration planning method are proposed. We make the following conclusions: (1) By studying the singularity of four-UPU parallel pouring mechanism, we propose the GA-IPS algorithm with angle observer, position observer, speed corrector and flexible control points. The numerical example shows the feasibility of crossing the singular region and correctness of the algorithm. (2) Based on motion characteristics of the high-speed cam and sloshing characteristics of the pouring liquid, the method of acceleration planning is studied. In view of the obvious characteristics of the impact vibration of the pouring liquid, the feasibility of piecewise acceleration planning method under the constraints of speed, acceleration and jerk is proposed and verified. (3) Based on the single pendulum model, the damping pendulum model the rotation and moving fusion of the pouring liquid under small sloshing condition is established, and fitting with the piecewise acceleration model, the maximum value of acceleration in each stage is given by the search method. The method of fitting the rotational acceleration is given by means of the relationship between the path and tilting angle. The experimental results also verify the correctness of the established single pendulum model and acceleration model.

1. Kozuszek, K., Robotic problem solving. Mod. Cast., 2006, 96(4), 24-28.

2. Terashima, K., Recent automatic pouring and transfer system in foundries. Sokeizai, 1998, 39, 1-8.

3. Wang, C., Li, L., Guo, Y. and Sheng, Y., Hybrid trussed mobile heavy duty casting robot, China Patent 201710-682226.8, 2017.

4. Chettibi, T., Lehtihet, H. E., Haddad, M. and Hanchi, S., Minimum cost trajectory planning for industrial robots. Eur. J. Mech., 2004, 23(4), 40703-40715.

5. Constantinescu, D. and Croft, E. A., Smooth and time-optimal trajectory planning for industrial manipulators along specified paths. J. Rob. Syst., 2000, 17(5), 233-249.

6. Park, J. and Bobrow, J. E., Reliable computation of minimum-time motions for manipulators moving in obstacle fields using a successive search for minimum-overload trajectories. J. Field Robot., 2005, 22(1), 1-14.

7. Dasgupta, B., Singularity-free planning for the Stewart platform manipulator. Mech. Mach. Theory, 1998, 33, 771-725.

8. Abdellatif, H. and Heimann, B., Adapted time-optimal trajectory planning for parallel manipulators with full dynamic modeling. In Proceedings of the 2005 IEEE International Conference on Robotics and Automotion, Barcelona, Spain, 2005.

9. Pugazhenthi, S., Nagarajan, T. and Singaperumal, M., Optimal trajectory planning for a hexapod machine tool during contour machining. Proc. Inst. Mech. Eng., Part C, 2002, 216(12), 12471257. 
10. Sen, S., Dasgupta, B. and Mallik, A. K., Variational approach for singularity-free path-planning of parallel manipulators. Mech. Mach. Theory, 2003, 38(11), 1165-1183.

11. Afroun, M., Chettibi, T. and Hanchi, S., Planning optimal motions for a DELTA parallel robot. In Proceedings of the 14th Mediterranean Conference on Control and Automation, Ancona, Italy, 2006.

12. Terashima, K., Kaneshige, A., Koyamatsu, H., Yamaura, K., Ronda, W. Y. and Inagaki, T., Modeling and motion control of fluid behavior in automatic pouring system. IFAC Proc. Vol., 1996, 29(1), 6197-6202.

13. Terashima, K., Hamaguchi, M. and Yamaura, K., Modeling and input shaping control of liquid vibration for an automatic pouring system. In Proceedings of 35th IEEE Conference on Decision and Control, Kobe, Japan, 1996.

14. Hamaguchi, M., Yamamoto, M. and Terashima, K., Modeling and control of sloshing with swirling in a cylindrical container during a curved path transfer. In Proceedings of the 2nd Asian Control Conference, Tokyo, Japan, 1997.

15. Yamagata, H. and Kaneko, S., Sloshing suppression control of contained liquid in a moving cylindrical container. Trans. JSME, 1997, 64(621), 671-674.

16. Yano, K. I. and Terashima, K., Robust liquid container transfer control for complete sloshing suppression. IEEE Trans. Control Syst. Technol., 2001, 9(3), 483-493.
17. Li, L., Wang, C. and Wu, H., Research on kinematics and pouring law of a mobile heavy load pouring robot. Math. Prob. Eng., 2018, 2018, 1-13.

18. Pan, $\mathrm{X}$. and $\mathrm{Xu}, \mathrm{Y}$. Figures selecting the basin in investment casting. Special-Cast Non-ferrous Alloys, 1996, 5, 34-36.

19. Binjie, M. and Hong, L., Analysis of harmonic response of liquid sloshing. Missiles Space Vehic., 2005, 1, 008.

20. Chen, J., Zhu, H., Zhang, L. and Sun, Y., Research on fuzzy control of path tracking for underwater vehicle based on genetic algorithm optimization. Ocean Eng., 2018, 156, 217-223.

21. Zhang, C. (ed.), High-speed CAM mechanism commonly used movement law. In Mechanical Dynamics, Higher Education Press, China, 2008, pp. 137-143.

ACKNOWLEDGEMENTS. We thank the anonymous reviewers for their valuable comments and suggestions. This work was supported by the Major Science and Technology Projects of Anhui Province of China (No. 16030901012) and the Postgraduate Research and Practice Innovation Program of Jiangsu Province (No. KYCX18_0260).

Received 12 September 2018; revised accepted 10 January 2019

doi: $10.18520 / \mathrm{cs} / \mathrm{v} 116 / \mathrm{i} 11 / 1829-1839$ 